\title{
CUIDADOS DE ENFERMAGEM E SEGURANÇA DO PACIENTE: REFLEXÃO SOBRE O TEMA E SUAS DIMENSÕES NO AMBIENTE DE UNIDADE DE TERAPIA INTENSIVA.
}

\author{
Charliene Pinto de Melo ${ }^{1}$, Chennyfer Dobbins Abi Rached ${ }^{2}$ \\ 1 Especialista em Terapia Intensiva. Faculdade Unyleya. Brasilia - Brasil \\ 2 Doutora em Saúde Coletiva; Mestre em Economia da Saúde pela Universidade \\ Federal de São Paulo (UNIFESP); Docente do Programa de Mestrado Profissional em \\ Gestão em Sistemas de Saúde - Universidade Nove de Julho - UNINOVE. São Paulo - \\ Brasil email: chennyferr@yahoo.com.br ORCID: https://orcid.org/0000-0002-4499- \\ 3716
}

\section{RESUMO}

A preocupação com qualidade do cuidado e com a segurança do paciente, atualmente, nas instituições de saúde, tem sido foco central das discussões, perpetuando um tema de discussão em âmbito mundial. Foram evidenciadas as seguintes fragilidades com a pressão para que o profissional da Saúde produza mais em empresas privadas, em tempo mais curto, para reduzir custos, e as superlotações de serviços de emergência, a posição corporativista dos profissionais, a organização da estrutura com alta variabilidade, a cobrança assistencial, a escassa padronização dos processos, ausência de liderança autêntica, falta de indicadores, falta de comunicação e de cultura de segurança e, ainda a formação falha sobre o assunto, todos este fatores isolados ou concomitantes geram condições de trabalho propício a ocorrência de eventos adversos. Palavras-chave: Segurança do Paciente, Unidade de Terapia Intensiva, Gestão em Saúde

\section{ABSTRACT}

The concern with quality of care and patient safety, currently in health institutions, has been the central focus of the discussions, perpetuating a worldwide discussion topic. The following weaknesses were evidenced with the pressure for the health professional to produce more in private companies, in a shorter time, to reduce costs, and overcrowding of emergency services, the corporatist position of professionals, organization of the structure with high variability, scarce standardization of processes, absence of authentic leadership, lack of indicators, lack of communication and safety culture, and lack of training on the subject, all these isolated or concomitant factors generate working conditions conducive to occurrence of adverse events.

Key words: Patient Safety, Intensive Care Unit, Health Management 


\section{INTRODUÇÃO}

A cada ano a OMS estima que milhões de pacientes em todo o mundo sofrem lesões incapacitantes ou morte devido a práticas médicas inseguras. Estima-se que, nos países ricos e tecnologicamente avançados, um em cada 10 pacientes é prejudicado ao receber cuidados médicos no ambiente hospitalar (WHO, 2008). A segurança do paciente (SP) representa um problema global de saúde pública que afeta os países em todos os níveis de desenvolvimento. A rede de SP foi criada em 2004 pela Organização Mundial da Saúde (OMS), para mobilizar esforços globais e melhorar a segurança dos cuidados de saúde para os pacientes atendidos no mundo inteiro (WHO, 2008).

A Unidade de Terapia Intensiva (UTI) é dotada de um sistema de monitorização contínua, que atende pacientes em estado potencialmente grave ou com descompensação de um ou mais sistemas orgânicos, oferece um suporte maior para pacientes e tem por objetivo a manutenção da vida e a recuperação da saúde, e para isso, utiliza se de intervenções complexas, tecnologia avançada e diversos dispositivos, aumentando assim as chances de erros e eventos adversos que colocam em risco a

segurança do paciente. É considerado um ambiente onde existem pacientes gravemente enfermos, que necessitam de aparelhos de tecnologia avançada, inúmeros dispositivos terapêuticos e, em alguns casos, manipulação excessiva, é plausível a afirmativa de que exista uma maior propensão à ocorrência de erros (BECCARIA et al., 2012).

A assistência à saúde isenta de riscos e falhas ao paciente é um objetivo a ser atingido pelos profissionais da saúde e um compromisso da formação profisssional (PADILHA, 2010). Acredita-se que, a assistência à saúde livre de riscos e danos sempre foi um requisito primordial para a manutenção da qualidade dos serviços prestados pelas instituições hospitalares, sejam elas públicas ou privadas. No entanto, o processo de cura pode estar acompanhado de efeitos indesejados e, algumas vezes, pode ser mais doloroso e sofrível que a própria doença (VINCENT, 2010).

Evidências mostram que quanto mais complexo o cuidado, maior será o avanço tecnológico, e associado a fatores que prejudicam o alcance da qualidade, por exemplo, o aperfeiçoamento pessoal ineficiente, gestão inadequada, negligência por parte dos profissionais, desmotivação, sobrecarga de trabalho, baixa remuneração, ausência ou limitação da sistematização e documentação do cuidado de enfermagem podem induzir ao erro e consequentemente aos danos (TOFFOLETTO, 2008).

A ocorrência de eventos iatrogênicos na assistência coloca em risco a vida de pacientes e tem merecido atenção dos enfermeiros na busca por cuidados que assegurem um mínimo de riscos (PESSALACIA et al., 2012). As investigações sobre práticas 
seguras preocupam os enfermeiros pois as pesquisas ainda não indicam abordagem específica aos desafios sobre segurança em enfermagem (ALVES, 2013).

Dentre as profissões da área da saúde, a enfermagem está na linha de frente quando trata-se da segurança, pois está constantemente envolvida com o cuidado e proteção dos pacientes, realiza à assistência direta ao paciente, acarretando a responsabilidade para manutenção de sua segurança. Em contrapartida, temos o despreparo e a falta de conhecimento de muitos profissionais, que acarreta em possibilidades de falhas na assistência e possíveis riscos à segurança do paciente, tornando-se um tema de grande discursão e um grande desafio para área assistencial.

Portanto, a cultura de SP está sendo construída em todo o mundo e se faz necessária a consolidação de uma política dentro dos hospitais, onde os erros sejam investigados e tratados de uma maneira não punitiva, restrita ao indivíduo e entendê-lo como um processo sistêmico, que permita a criação de estratégias de segurança que previnam ocorrências indesejáveis. (WACHTER, 2010).

Oferecer uma assistência livre de danos é um compromisso que deve ser tomado pelos profissionais de saúde. No entanto, é correto admitir que o ser humano cometa erros e, para assumir a prestação de um cuidado seguro, é preciso identificar o erro e avaliar os fatores relacionados para que se possam criar medidas que visem preveni-los e aprimorar a assistência (HARADA et al., 2006).

Qual o conhecimento produzido sobre segurança do paciente no gerenciamento de enfermagem evidenciados nas produções científicas das bases virtuais?

A escolha pela discussão sobre Segurança do Paciente tem como fundamento minha vivência com o universo da terapia intensiva e meu vislumbramento com o papel que o enfermagem nesse cenário de cuidados a pacientes críticos. Por meio da vivência diária e da rotina de uma Unidade de Terapia Intensiva, identifiquei a importância do tema e o papel da enfermagem neste contexto, identifiquei a necessidade de um plano de cuidados específico para segurança do paciente, elaboração de estratégias e indicadores definidos para avaliação da segurança.

A OMS aponta que a segurança dos pacientes é um princípio fundamental dos cuidados de saúde, pois cada etapa do processo de prestação de cuidados possui certo grau de insegurança inerente. A partir disso, surgem diversos questionamentos sobre medidas que devem ser tomadas a fim de reduzir ao máximo possível os riscos enfrentados por quem precisa de cuidados médicos e hospitalares (WHO, 2008). 
O presente trabalho visa analisar o conhecimento dos profissionais de enfermagem a cerca da segurança do paciente, tendo em vista que a equipe de enfermagem representa praticamente $60 \%$ da força de trabalho em saúde, não há dúvidas de seu papel fundamental nos processos que envolvem a atenção ao paciente. É vital que a categoria lance um novo olhar sobre suas práticas cotidianas e identifique possíveis falhas no processo de gerar erros. Sem dúvida, os profissionais de enfermagem podem fazer muito para alcançar o cuidado seguro.

\section{Objetivos}

\section{Objetivo Geral:}

- Refletir sobre o conceito de Segurança do paciente e suas dimensões, no contexto da equipe de Enfermagem.

\section{Objetivos Específicos:}

- Evidenciar o conhecimento produzido acerca da temática a contribuição da enfermagem para a segurança do paciente;

- Identificar os cuidados de enfermagem realizados em relação a segurança do paciente em Unidades de Terapia Intensiva;

- Refletir sobre a contribuição da enfermagem para a segurança do paciente na construção do cuidado seguro;

- Correlacionar a produção de conhecimento sobre segurança do paciente e eventos adversos com as metas internacionais e identificar possíveis inovações tecnológicas em segurança do paciente no gerenciamento de enfermagem;

- Apontar os principais eventos adversos/erros que são cometidos na UTI e as medidas recomendadas para os profissionais da enfermagem que visam garantir a segurança do paciente em UTI.

\section{METODOLOGIA}

Trata-se de uma pesquisa de revisão bibliográfica da literatura, com abordagem qualitativa. 
A coleta de dados será realizada pelas bases de dados Lilacs, Medline, BDENF, SciELO e PubMed por meio dos descritores 'segurança do paciente', 'cuidados de enfermagem' e 'UTI' 'unidade de terapia intensiva' 'Brasil'.

Os artigos selecionados serão referem-se ao período 2010-2018, disponíveis em português, inglês e espanhol.

\section{REFERENCIAL TEÓRICO}

\section{Segurança do Paciente}

A segurança do paciente é um importante tema para todos os profissionais da área da saúde, pois exibe uma preocupação com novas tecnologias e saberes que possam colaborar para a prática clínica, assegurando assim a qualidade na assistência prestada. Podendo ser definida em sua forma mais simples como o ato de evitar, prevenir e melhorar os resultados adversos ou as lesões originadas no processo de atendimento médico-hospitalar.

Observa-se uma dificuldade de estabelecer uma divisão entre a segurança do paciente e atividades relacionadas, como gerenciamento do risco e garantia da qualidade (VICENT, 2009).

No âmbito da saúde observa um grande movimento pela qualidade da assistência, temática que crescimento relacionado à conscientização e representa um compromisso ético, moral e social dos envolvidos em questão. Para garantir a segurança, deve-se verificar a melhor maneira de gerenciar os aspectos envolvidos, controlar e diminuir a ocorrência de eventos adversos (BEZERRA et al, 2009). Sob essa perspectiva, muitas pesquisas estão sendo realizadas, para verificar a incidência, as causas, medidas preventivas, os fatores relacionados e a percepção dos profissionais frente ao tema.

Segundo a Agência Nacional de Vigilância Sanitária a qualidade dos atendimentos na área da saúde é crucial para reduzir os danos e controlar os riscos. Dessa forma um conjunto de ações são necessárias para identificação da ocorrência de eventos precoces que afetam a segurança do paciente, reduzir danos e evitar riscos futuros.

No Brasil diversas iniciativas foram criadas com o intuito promover a segurança do paciente. Os órgãos e serviços responsáveis por transfusões de sangue, pelo controle de infecções hospitalares e pelos serviços de anestesia, são pioneiros ao se tratar de medidas de prevenção que garantam a qualidade assistencial. Em 2002, destaca-se a Rede Sentinela. Os serviços que compõem a Rede monitoram e notificam eventos 
adversos e queixas técnicas provenientes de produtos sob vigilância sanitária, como medicamentos, vacinas e imunoglobulinas; pesquisas clínicas; cosméticos, produtos de higiene pessoal ou perfume; artigos e equipamentos médico-hospitalares; kit reagente para diagnóstico in vitro; uso de sangue ou componentes; saneantes e agrotóxicos, em uso no Brasil, fazendo a Vigipós dos produtos utilizados nos estabelecimentos da área da Saúde (ibid) (BRASIL, 2013).

Outros programas e políticas do Ministério da Saúde, em parceria com as Secretarias Estaduais e Municipais de Saúde, contribuem com o PNSP. Merecem destaque: o conjunto de iniciativas da Agência Nacional de Vigilância Sanitária (Anvisa), em especial o programa hospital sentinela; o Programa Nacional de Avaliação de Serviços de Saúde (PNASS); o Programa de Apoio ao Desenvolvimento Institucional do Sistema Único de Saúde (Proadi-SUS); a parceria entre o Ministério da Saúde (MS) e as entidades de Saúde detentoras do Certificado de Entidade Beneficente de Assistência Social em Saúde (Cebas-Saúde); o Projeto de Formação e Melhoria da Qualidade da Rede de Atenção à Saúde (QualiSUS-Rede); a Política Nacional de Humanização (PNH); o processo de certificação dos Hospitais de Ensino, sob a coordenação do Ministério da Saúde e do Ministério da Educação, a Política Nacional de Segurança e Saúde no Trabalho (PNSST - Decreto $n^{0} 7.602$ de 7 de novembro de 2011) e a Política Nacional de Atenção Hospitalar (PNHOSP), que estabelece as diretrizes para a reorganização do componente hospitalar da Rede de Atenção à Saúde, recentemente pactuada na comissão intergestora tripartite.

Além dessas medidas, as categorias da área da saúde se organizam também para minimizar esses riscos e discutir a implantação de estratégias para melhorar a Segurança do Paciente. Destacam-se entre elas a Rede Brasileira de Enfermagem e Segurança do Paciente (Rebraensp) e a Sociedade Brasileira de Enfermagem em Feridas e Estética (Sobenfee) (ibid).

A World Alliance for PatientSafety (Aliança Mundial para a Segurança do Paciente) foi criada em 2004 pela Organização Mundial de Saúde (OMS). O Brasil é um dos países que a compõe. Desde sua criação, com objetivos como organizar conceitos e definições sobre o tema, tem elaborado programas e diretrizes que visam mobilizar e sensibilizar profissionais da área da saúde e a população para busca de soluções que promovam a segurança do paciente, divulgando conhecimentos e desenvolvendo estratégias para a mudança do cenário mundial (AVELAR et al, 2010). 


\section{A segurança do paciente de acordo com as metas internacionais para segurança do paciente}

A Joint Commission International (JCI), em parceria com a Organização Mundial da Saúde lançou seis metas para direcionar as ações importantes na assistência ao paciente, sendo elas:

Meta 1: identificar corretamente os pacientes;

Meta 2: melhorar a comunicação entre profissionais de saúde;

Meta 3: melhorar a segurança na prescrição, no uso e na administração de medicamentos;

Meta 4: assegurar cirurgia em local de intervenção, procedimento e paciente corretos;

Meta 5: reduzir o risco de infecção associado aos cuidados de saúde;

Meta 6: reduzir os riscos de lesões provenientes de quedas e úlceras por pressão.

O objetivo dessas metas é promover melhorias específicas na segurança do paciente por meio de estratégias que abordam aspectos problemáticos na assistência a saúde, apresentando soluções baseadas em evidências para esses problemas.

\section{PRINCIPAIS EVENTOS ADVERSOS/INCIDENTES E ERROS QUE OCORREM NA UTI}

Embora o fator humano esteja presente nos eventos adversos, condições de trabalho, aspectos estruturais e atividades desenvolvidas podem estar relacionadas ao desencadeamento de erros (PADILHA, 2006).

A ocorrência de evento adverso representa um sério problema em unidade de terapia intensiva e pode impactar em diversos processos como aumento do tempo de internação e na mortalidade. Uma questão fundamental para a melhoria da qualidade e segurança do paciente é determinar a magnitude de eventos adversos, o perfil de pacientes e os fatores associados à ocorrência de dano decorrente da prestação de cuidados de saúde. Se faz necessário também fortalecer a cultura de segurança a fim de intervir no processo assistencial e estabelecer um compromisso com a segurança de todos os envolvidos.

Para que seja possível pontuar os principais eventos adversos, se faz necessário primeiramente dar uma definição para o termo, diversos autores propõe está definição, dentre eles: Eventos adversos (EAs) são definidos como complicações não desejadas provenientes da assistência prestada aos pacientes, não associadas à evolução natural da doença de base, causando lesões mensuráveis e/ou prolongamento da internação 
(GALLOTTI, 2004). A OMS (2009), definiu evento adverso como um incidente que resulta em algum dano ao indivíduo com implicações no comprometimento da estrutura e das funções corporais e/ou quaisquer efeitos nocivos decorrentes de lesão, sofrimento, incapacidade ou morte, podendo ser físico, social ou psicológico.

Vários termos são utilizados como sinônimos de eventos adversos: ocorrências de eventos iatrogênicos; erros de enfermagem; ocorrências, reações; complicações iatrogênicas; iatrogênicas; doença iatrogênica e falhas. Entretanto, todas são definidas como eventos indesejáveis, não intencionais, de natureza danosa ou prejudicial ao paciente, comprometendo sua segurança, consequente ou não de falha do profissional envolvido (SILVA, 2003).

Verifica-se que a prática insegura ainda tende a persistir nos processos assistenciais e administrativos dos estabelecimentos de saúde em todo o mundo. A Organização Mundial de Saúde (OMS) estima que danos à saúde ocorram em dezenas de milhares de pessoas todos os anos no mundo. Dados do Instituto de Medicina (IOM) dos Estados Unidos da América (EUA) indicam que erros associados à assistência à saúde causam entre 44.000 e 98.000 disfunções a cada ano nos hospitais dos EUA. Estudo realizado em 2013 com o objetivo de atualizar esses números demonstrou que a estimativa de mortes prematuras associadas a danos evitáveis decorrentes ao cuidado hospitalar estaria entre 210.000 e 400.000 americanos por ano. Estudos recentes mostram que a incidência de Eventos Adversos (EA) no Brasil é alta. A ocorrência deste tipo de incidente no país é de 7,6\%, dos quais $66 \%$ são considerados evitáveis.

Conforme Bohomol e Ramos (2007), os erros podem trazer prejuízos diversos aos pacientes, desde o aumento de sua permanência em um ambiente hospitalar, necessidade de intervenção diagnóstica e terapêutica, até mesmo a morte. Além de danos ao cliente, há também, aspectos econômicos, como aumentos dos custos das internações hospitalares.

Conforme Paiva e Berti (2010) os boletins de notificação de eventos adversos são importantes fontes de alerta e informação, promovendo a segurança no ambiente hospitalar e contribuindo para o gerenciamento da assistência de enfermagem.

É imprescindível para a segurança do paciente e a contínua melhoria no cuidado à saúde que haja uma revelação, notificação e discussão dos erros ocorridos, como um procedimento padrão nas instituições. Somente através de discussões apropriadas e atitudes claras as discussões dos erros poderão ser identificadas.

Estudar os erros é vital para às mudanças na prática que levarão à prevenção destes no futuro (KALRA, 2004). 
É de suma importância do registro e das notificações dos eventos adversos para que se desenvolva tecnologias referentes aos mesmos, deve-se entender a prevalência deles, bem como seus tipos e outros aspectos, como já dito anteriormente nessa discussão.

Os eventos adversos na assistência de enfermagem podem ser categorizados da seguinte forma: Eventos adversos relacionados à administração de medicamentos; Eventos adversos relacionados à vigilância do paciente; Eventos adversos relacionados à manutenção da integridade cutânea; Eventos adversos relacionados aos recursos materiais (Duarte SCM, et al. 2015).

- Eventos adversos relacionados à administração de medicamentos

Os eventos adversos associados à administração de medicamentos são os mais comuns, e a probabilidade de um indivíduo sobreviver a uma internação hospitalar livre da ocorrência destes eventos poderá variar de acordo com o tempo.

$\checkmark$ Omissão de medicamentos;

$\checkmark$ Erros no preparo do medicamento;

$\checkmark$ Erros no horário de administração;

$\checkmark$ Dose do medicamento inadequada e erros na técnica de administração.

- Eventos adversos relacionados à vigilância do paciente

$\checkmark$ Queda do paciente do leito e da própria altura;

$\checkmark$ Perda de cateteres, sondas e drenos

- Eventos adversos relacionados à manutenção da integridade cutânea

$\checkmark$ Não realização de mudança de decúbito;

$\checkmark$ Posicionamento inadequado do paciente no leito.

- Eventos adversos relacionados aos recursos materiais

$\checkmark$ Falta de equipamentos;

$\checkmark$ Equipamentos com defeito.

Para uma assistência de qualidade e livre de danos e agravos, se faz necessário medidas preventivas e de fiscalização continuas. Educação continuada de toda equipe e realização de indicadores periódicos ajudam a melhoria da assistência prestada. 


\section{O CONHECIMENTO PRODUZIDO ACERCA DA TEMÁTICA A CONTRIBUIÇÃO DA ENFERMAGEM PARA A SEGURANÇA DO PACIENTE}

A meta oferecer assistência de excelência, de qualidade, no âmbito da saúde tem sido cada vez mais valorizada, e para que seja possível atingir este padrão faz se necessário uma equipe comprometida e empenhada.

Oferecer uma assistência livre de danos é um compromisso que deve ser tomado pelos profissionais de saúde. No entanto, é correto admitir que o ser humano cometa erros e, para assumir a prestação de um cuidado seguro, é preciso identificar o erro e avaliar os fatores relacionados para que se possam criar medidas que visem preveni-los e aprimorar a assistência (HARADA et al., 2006).

A proteção ao paciente oferecida por meio de uma assistência segura é responsabilidade de cada profissional, sendo impulsionada também pela maior exigência da sociedade (PADILHA, 2001).

Apesar dos avanços na área de saúde, a segurança do paciente é influenciada pelas iatrogenias cometidas pelos profissionais, as quais refletem diretamente na qualidade de vida dos clientes, provocando consequências desagradáveis tanto para os pacientes como para os profissionais e para a organização hospitalar (MIASSO et al., 2006).

O enfermeiro gerencia o cuidado, está ação implica diretamente na qualidade e na excelência da assistência oferecidas a clientela. O serviço de enfermagem, deve promover meios que facilitem a comunicação destes eventos e a captação das informações necessárias a fim de visar a promoção da segurança do paciente no ambiente hospitalar e estabelecer mecanismos para a prevenção de eventos adversos e minimização de erros, (PAIVA et al, 2010).

Os profissionais de enfermagem são responsáveis por grande parte das ações assistenciais encontrando-se assim em posição privilegiada para reduzir a possibilidade de incidentes que atingem o paciente, além de detectar as complicações precocemente e realizar as condutas necessárias para prevenção de agravos.

A enfermagem tem como objetivo principal cuidar da saúde e bem-estar do indivíduo, família e comunidade, agindo na promoção, proteção, recuperação e reabilitação da saúde, respeitando princípios éticos e legais no cuidar do ser humano (PIZZOLI LML, 2016)

O enfermeiro deve responsabilizar-se pelo planejamento das ações de enfermagem no tocante à disponibilização de recursos materiais adequados e seguros, como também na capacitação da equipe e promoção de condições tanto de trabalho 
como ambientais adequadas para a realização do cuidado, garantindo a segurança para o paciente (BOHOMOL E, RAMOS LH. 2003)

A qualidade do cuidado de enfermagem precisa ser continuamente avaliada, tanto para aprimorar a assistência, como para entender e refletir sobre a segurança do cuidado ofertado (VITURI DW, MATSUDA M. 2009).

A enfermagem e as instituições de saúde devem criar uma cultura de segurança, com enfoque na eficiência, qualidade, realizando gestão ativa e participativa, promoção da participação dos profissionais nas decisões referentes a desenho e fluxo dos processos de trabalho e a implementação de cultura de aprendizado constante em todas as questões vinculadas ao processo de trabalho (MENDES, et al 2005).

O cuidado seguro resulta tanto de ações corretas dos profissionais de saúde, como de processos e sistemas adequados nas instituições e serviços, assim como de políticas governamentais regulatórias, exigindo um esforço coordenado e permanente. A preocupação com a segurança já se mostra implícita no modelo brasileiro de atenção à saúde, que é “pautado na defesa da vida (MINISTÉRIO DA SAÚDE, 2010)

Para que o cuidado seja seguro, também é necessário construir uma cultura de segurança do paciente, em que profissionais e serviços compartilhem práticas, valores, atitudes e comportamentos de redução do dano e promoção do cuidado seguro. É preciso que medidas de segurança sejam sistematicamente inseridas em todos os processos de cuidado (Kohn LT, Corrigan JM, Donaldson MS, 2000)

$\mathrm{O}$ conceito de cultura de segurança tem sua origem em outras áreas, como aviação e energia nuclear, nas quais o trabalho se caracteriza pela complexidade e pelo risco. $\mathrm{Na}$ área da saúde, é um conceito ainda em construção, mas alguns de seus aspectos básicos já estão identificados (Agency for Healthcare Research and Quality AHRQ, 2013)

A pressão para que o profissional da Saúde produza mais em empresas privadas, em tempo mais curto, para reduzir custos, e as superlotações de serviços de emergência do SUS são exemplos bastante corriqueiros neste País de condições de trabalho que causam intenso sofrimento aos profissionais da Saúde e podem ser responsáveis por eventos adversos (BRASIL, 2014).

Estudo de Bartalha (2010) sinaliza que o reconhecimento dos problemas de segurança do paciente nas instituições de saúde é o primeiro passo para a realização de melhorias nesta área, já que é através da identificação dos problemas que pode-se analisar suas causas, consequências e encontrar diferentes maneiras para solucioná-los. 
Outra maneira efetiva no auxílio a melhoria do cuidado prestado aos pacientes no ambiente hospitalar é a correta utilização dos sistemas de informação.

Nesse sentido os sistemas informatizados podem ser uma estratégia de melhoria da segurança tal como é demonstrado em pesquisa realizada em quatro instituições que identificou ganhos na prática clínica com a adoção do prontuário eletrônico como redução dos erros de medicação, redução da utilização de recursos desnecessários ou inapropriados ao cuidado, aumento a aderência aos protocolos, monitorização das doenças crônicas e de seus cuidados e melhora na capacidade de aumentar a sobrevida (CHAUDHRY et al., 2006).

\section{CONTRIBUIÇÃO DA ENFERMAGEM PARA A SEGURANÇA DO PACIENTE NO BRASIL}

Garantir a segurança de todos que utilizam os serviços de saúde é um dos mais importantes desafios que o cuidado em saúde enfrenta, atualmente (Department of Health, 2004). A identificação, análise e gerenciamento de riscos, relacionados aos incidentes de segurança, são necessários para alcançar cuidado mais seguro e minimizar os danos (World Health Organization, 2008). Evidenciando assim que as ações dos enfermeiros podem contribuir para prevenir e evitar erros, pois a essência da Enfermagem é o cuidado (RADÜNZ V, 1999), os enfermeiros devem defender as políticas de saúde e enfermagem que assegurem acesso ao tratamento de qualidade, incluindo a administração segura de medicamentos.

O Código de Ética dos Enfermeiros leva em consideração as necessidades da população e os direitos ao cuidado de enfermagem, focado na pessoa, família e coletividade, e assume que os enfermeiros, em conjunto com os pacientes, defendam o cuidado à saúde, livre de riscos preveníveis e danos, e acessível a toda a população(COREN-SC, 2007).

A melhoria da segurança do cuidado em saúde reduz as doenças e danos, diminui o tratamento e/ou o tempo de hospitalização, melhora ou mantém o status funcional do paciente, e aumenta sua sensação de bem-estar(OMS, 2002).

Entretanto, mesmo com as iniciativas das instituições e dos representantes mundiais, existe evidência inadequada sobre o melhor caminho para alcançar a segurança de medicação, nos complexos sistemas de saúde. No contexto hospitalar, muitos fatores podem provocar danos aos pacientes. $\mathrm{O}$ ambiente do cliente envolve muitos fatores físicos, psicológicos, culturais, entre outros, que influenciam ou afetam a sobrevivência (Constatinou E, et al, 2004; OMS, 2002). Uma vez que os profissionais 
são responsáveis pelo planejamento e intervenção apropriada com a finalidade de manter ambiente seguro, é vital o desenvolvimento de pesquisa em enfermagem sobre segurança de medicação.

\section{CONSIDERAÇÕES FINAIS}

A partir do trabalho realizado constatou-se que o tema "Segurança do Paciente" é uma questão que vem sendo discutida mundialmente. É o primeiro pilar da qualidade na assistência à saúde, e não é possível oferecer uma boa assistência à saúde sem que seja oferecido segurança.

Foram evidenciadas as seguintes fragilidades com a pressão para que o profissional da Saúde produza mais em empresas privadas, em tempo mais curto, para reduzir custos, e as superlotações de serviços de emergência, a posição corporativista dos profissionais, a organização da estrutura com alta variabilidade, a cobrança assistencial, a escassa padronização dos processos, ausência de liderança autêntica, falta de indicadores, falta de comunicação e de cultura de segurança e, ainda a formação falha sobre o assunto, todos este fatores isolados ou concomitantes geram condições de trabalho propício a ocorrência de eventos adversos.

Por meio da análise das percepções dos trabalhos analisados é necessário fomentar a implementação e aperfeiçoamento das práticas de segurança do paciente, a partir de estratégias para a redução ao mínimo dos riscos ao cuidado em saúde. Para que a segurança do paciente seja realmente efetiva, é necessário esforço e comprometimento diário de equipes multiprofissionais, instituições e serviços de atenção à saúde.

Pretendo deixar com o presente trabalho um panorama geral atualizado sobre a temática, com o intuito de subsidiar novas práticas e aprimorar o que é realizado, com adoção de novas medidas com base nos estudos que contemplem essa temática.

\section{REFERÊNCIAS BIBLIOGRÁFICAS}

ALVES E. F. O Cuidador de Enfermagem e o cuidar em uma unidade de terapia intensiva. Rev Cient Ciênc Biol Saúde. 2013;15(2):115-22.

BARBOSA T. P. et al. Práticas assistenciais para segurança do paciente em unidade de terapia intensiva. Acta Paul Enferm. 2014; 27(3):243-8.

BECCARIA, L. M. et al. Eventos adversos na assistência de enfermagem Rev. Bras. Ter. Intensiva, v. 21, n. 3, p. 276-282, 2009. Disponível em: $<$ http://www.scielo.br/pdf/rbti/v21n3/a07v21n3> Acesso em: 10 jan.2012 
HARADA, M.J.C.S. et al. O erro humano e a segurança do paciente. São Paulo: Atheneu; 2006.

PADILHA K. G. et al. Ocorrências iatrogênicas com medicação em unidade de terapia intensiva: condutas adotadas e sentimentos expressos pelos enfermeiros. Rev Escola Enferm USP. 2010;36(1):50-7.

PESSALACIA J. D. et al. Atuação da equipe de enfermagem em UTI pediátrica: um enfoque na humanização. Rev Enferm Cent O Min. 2012;2(3):410-8.

TOFFOLETTO, M. C. Fatores associados aos eventos adversos em uma Unidade de Terapia Intensiva. Tese (Doutorado). Escola de Enfermagem. Universidade de São Paulo. São Paulo, 2008.

VINCENT, C. Segurança do paciente: orientações para evitar eventos adversos. 1. ed. São Caetano do Sul, SP: Yendis, 2010.

WACHTER, R.M. Compreendendo a segurança do paciente. Porto Alegre (RS): Artmed; 2010.

WHO - World Health Organization/World Alliance for Patient Safety. Summary of the evidence on patient safety: implications for research. The Research Priority Setting Working Group of the World Alliance for Patient Safety. Geneva: World Health Organization, 2008.

KOHN LT, CORRIGAN JM, DONALDSON MS, EDITORS. To err is human: building a safer health system. Washington, DC (US): National Academy Press; 2000.

AGENCY FOR HEALTHCARE RESEARCH AND QUALITY - AHRQ (US). Safety culture. Rockville, MD; [data desconhecida] [acesso em 2013 Mar 5]. Disponível em: http://psnet.ahrq.gov/primer.aspx?primerID $=5$

BARTALHA, E.M.S.S. A cultura de segurança do paciente na percepção de profissionais de enfermagem de um hospital universitário. 2012. 154 f. Dissertação (Mestrado) - Escola de Enfermagem da Universidade de São Paulo, Universidade de São Paulo, São Paulo, 2012.

BRASIL. Ministério da Saúde. Documento de referência para o Programa Nacional de Segurança do Paciente / Ministério da Saúde; Fundação Oswaldo Cruz; Agência Nacional de Vigilância Sanitária. - Brasília : Ministério da Saúde, 2014.40 p. : il. ISBN 978-85-334-2130-1

PIZZOLI LML. Qualidade de vida no trabalho: um estudo de caso das enfermeiras do Hospital de Heliópolis. Ciênc. Saúde Colet. 2005 [citado em 19 dez 2016]; 11(4):10652. DOI: http://dx.doi.org/10.1590/S1413- 81232005000400028. 
BOHOMOL E, RAMOS LH. Erros de medicação: causas e fatores desencadeantes sob a ótica da equipe de enfermagem. Acta Paul Enferm. 2003;16(2):41-8. Institute of Medicine of the National Academies. The Chasm in Quality: Select the Indicators from Recent Reports. Institute of Medicine [internet]. 2006 [acesso: 24 mai 2008]. Disponível em: http://www.iom.edu/?id=14991

JOINT COMMISSION FOR PATIENT SAFETY. World Alliance for PatientSafety [internet]. 2008 [acesso: 30 jul. 2009] Disponível em: http://www.ccforpatientsafety.org/30730/

ANVISA. Anvisa promove debate sobre segurança do paciente no Brasil. Ministério da Saúde. [internet]. 2007 [acesso: 15 mai. 2008]. Disponível em: http://www.anvisa.gov.br/DIVULGA/ noticias/2007/171007.htm

JOINT COMMISSION FOR PATIENT SAFETY. Nine Patient Safety Solutions [internet]. 2008 [acesso: 15 jun 2008]. Disponível em: http://www.ccforpatientsafety.org/30723/

JOINT COMMISSION FOR PATIENT SAFETY. High 5s Project [internet]. 2008 [acesso: 15 jun 2008]. Disponível em: http://www.ccforpatientsafety.org/30753/

DEPARTMENT OF HEALTH. About Patient Safety [internet]. 2004 [acesso: 20 jun 2008]. Disponível em: http://www.dh.gov.uk/en/Publichealth/Patientsafety/Patientsafetygeneralinformation/D H_4066328

WORLD HEALTH ORGANIZATION. 10 facts on patient safety [internet]. 2008 [acesso: 20 jun 2008]. Disponível em: http:// www.who.int/features/factfiles/patient_safety/en/index.html

RADÜNZ V. Cuidando e se cuidando: fortalecendo o self do cliente oncológico e o self da enfermeira. 2a ed. Goiânia: AB; 1999.

COREN-SC. Conselho Regional de Enfermagem de Santa Catarina. Código de Ética dos Profissionais de Enfermagem [internet]. 2007 [acesso: 05 jun 2008]. Disponível em: http://www.coren-sc.org.br/Empresa2/Cepreform.html

CONSTATINOU E, ROMANIUK D. CLIENT SAFETY. IN: POTTER P, PERRY A, ROSS-KERR, WOOD M. Canadian Fundamentals of Nursing. Toronto: Elsevier; 2004.

OMS. Organização Mundial da Saúde. 26a Conferência Sanitária Pan-americana 54a Sessão do Comitê Regional - Qualidade da Assistência: Segurança do Paciente. Organização Pan-Americana da Saúde [internet]. 2002 [acesso: 23 mai 2008] Disponível em:http://www.ops-oms.org/portuguese/gov/csp/csp26-26-p.pdf 
VINCENT, CHARLES. Segurança do Paciente: Orientações para evitar eventos adversos. São Caetano do Sul: Yendis, 2009.

BEZERRA, Ana Lúcia Queiroz et al. Análise de queixas técnicas e eventos adversos notificados em um hospital sentinela.Rev. enferm. UERJ, Rio de Janeiro, 2009 out/dez; 17(4):467-72.

GALLOTTI, Renata MahfuzDaud. Eventos adversos - o que são?RevAssocMedBras 2004; 50(2): 109-26.

ANVISA. RDC $\mathbf{N}^{\circ} \mathbf{2}$, de 25 de janeiro de 2010. Dispõe sobre o gerenciamento de tecnologias em saúde. Publicada no DOU de 26/01/2010. Brasília, 2010.

ANVISA. Ministério da Saúde. Segurança do Paciente e Qualidade em Serviços de Saúde. Hotsite. Disponível em: $<$ http://www.anvisa.gov.br/hotsite/segurancadopaciente/index.html $>$. Acesso em $10 \mathrm{de}$ março de 2014.

BRASIL. Agência Nacional de Vigilância Sanitária. Boletim informativo sobre segurança do paciente e qualidade em serviços de saúde. v. 1, n. 1, jan-jul, 2011. Brasília: GGTES/ Anvisa, 2011.

BRASIL. Ministério da Saúde. Assistência Segura: Uma Reflexão Teórica Aplicada à Prática. Agência Nacional de Vigilância Sanitária. Brasília: GGTES/Anvisa, 2013.

BRASIL. Ministério da Saúde. Documento de referência para o Programa Nacional de Segurança do Paciente. Agência Nacional de Vigilância Sanitária. Brasília: Ministério da Saúde, 2013.

BRASIL. Ministério da Saúde. Programa Nacional de Segurança do Paciente. Agência Nacional de Vigilância Sanitária. Brasília: Ministério da Saúde, 2013.64

BRASIL. Ministério da Saúde. Secretaria-Executiva. Área de Economia da Saúde e Desenvolvimento. Avaliação de Tecnologias em Saúde: Ferramentas para a Gestão do SUS. Brasília: Editora do Ministério da Saúde, 2009.

VITURI DW, Matsuda M. Content validation of quality indicators for nursing care evaluation. Rev. Esc. Enferm. da USP, São Paulo, v. 43, n. 2., p. 429-437, jun. 2009.

SANTOS BP, FERREIRA GB, SOARES MC, MEINCKE SMK, KÖNZGEN SM. Ensino de enfermagem no Brasil: do advento do sistema Nightingale ao cenário científico. Hist. Enf. Rev. Eletr. 2014 Aug.-Dec. [cited 2017 Jan 22]; 5(2):310- 22. Available from: http://pesquisa.bvs.br/brasil/resource/pt/bde-26779. 
